\title{
Application de l'optique intégré sur verre à la vélocimétrie laser Doppler pour la mesure de vitesse et de frottement en région pariétale
}

\author{
P. Lemaître-Auger ${ }^{1}$, S. Gluck ${ }^{2}$, Alain Cartellier ${ }^{3}$ et P. Benech ${ }^{1}$ \\ ${ }^{1}$ IMEP, UMR 5130 CNRS/INPG/UJF, 23 rue des Martyrs, BP 257, 38016 Grenoble Cedex 1 \\ 2 A2 Photonic Sensors, 16 rue des Cerisiers, 38800 Le Pont de Claix \\ ${ }^{3}$ LEGI, UJF-CNRS-INPG, 1025 rue de la Piscine, BP 95, 38042 St-Martin d'Hères cedex
}

\begin{abstract}
Un Vélocimètre Laser Doppler entièrement Intégré (VLDI) sur un substrat de verre est présenté dans cet article. Ce nouvel instrument d'investigation est dédié à la mesure de vitesses en proche paroi, dans la sous-couche visqueuse. De la mesure de vitesse faite par ce capteur se déduit directement le frottement entre le fluide et la paroi. Ce capteur peut également être utilisé en micro-fluidique. La tête du capteur est réalisée en une seule étape technologique par diffusion d'ions : Ag+ « Na+. Selon la géométrie choisie, la plaquette de verre de $1 \mathrm{~mm}$ d'épaisseur mesure quelques millimètres de large (entre 2 et $8 \mathrm{~mm}$ selon les cas) par 3 centimètres de long. La tête du VLDI est alimentée par une diode laser $(10=0,8 \mu \mathrm{m})$ via une fibre optique monomode. Les signaux Doppler quant à eux sont transmis du capteur vers une diode avalanche par une fibre optique multimode. Une des spécificités de ce capteur de deuxième génération est la production d'un petit volume de mesure : $18{ }^{\prime} 25 ' 10 \mu \mathrm{m} 3$ selon les axes $x, y$ et $z$ ( $x$ est orienté selon l'écoulement du fluide alors que y est la direction perpendiculaire à la paroi.) pour un interfrange de $0,83 \mu \mathrm{m}$ et de $18{ }^{\prime} 35^{\prime} 18$ pour un interfrange de $1,0 \mu \mathrm{m}$. Ces mesures sont prises à $1 / \mathrm{e} 2$ de l'intensité maximale présente dans le volume de mesure. La focalisation des faisceaux s'effectue grâce à l'intégration de miroirs convergent sur le substrat de verre.
\end{abstract}

Mots clés - Vélocimètre laser Doppler intégré, Optique intégrée, échange d'ions

\section{INTRODUCTION}

La mesure de la vitesse d'écoulement d'un fluide près d'une paroi et de la friction entre les deux n'est pas une tâche aisée. Différentes techniques, qu'elles soient intrusives ou non intrusives, sont possibles[1-4]. Parmi les techniques non intrusives, la vélocimétrie laser Doppler est très certainement la plus prometteuse. Le principe physique d'une telle sonde est le suivant. Il consiste à produire des franges linéaires d'interférence optique dans une région localisée de l'espace, appelée le volume de mesure. L'intensité lumineuse qui est diffusée par des particules contenues dans le fluide et passant à travers le volume de mesure est détectée avec un photodétecteur. Ceci donne lieu à un signal modulé temporellement à cause de la distribution spatiale de l'intensité optique dans le volume de mesure. La composante de la vitesse qui est perpendiculaire à l'axe optique est alors simplement le produit de cette fréquence, appelé la fréquence Doppler $\left(\mathrm{f}_{\mathrm{D}}\right)$, par l'interfrange $(\Delta \mathrm{x})$.

Toutefois, lorsque l'on utilise un vélocimètre laser Doppler classique, d'importantes difficultés bien identifiées existent. La première est la nécessité d'avoir une très grande résolution spatiale. Cette difficulté ne peut se résoudre que par un design optique complexe et volumineux qui sera sensible aux vibrations. Par exemple, des volumes compris entre $30 \times 20 \times 20 \mathrm{~mm}^{3}$ et $200 \times 200 \times 300 \mathrm{~mm}^{3}$ ont ainsi été obtenus[5-8]. La deuxième difficulté est la précision exigée sur la mesure de la distance entre le centre du volume de mesure et la paroi. La troisième grande difficulté est la limitation sur la valeur inférieure de cette distance. En effet, de façon générale, la diminution du rapport signal sur bruit des signaux Doppler causée par des réflexions parasites empêche d'approcher le volume de mesure de la paroi aussi près qu'on le voudrait[9,10]. Les plus petites distances que l'on trouve dans la littérature se situent entre 100 à $200 \mu \mathrm{m}[11-13]$.

Pour remédier à ces problèmes, nous proposons dans cet article un vélocimètre laser Doppler intégré de deuxième génération[14-15] spécialement conçu pour la mesure de vitesse dans la sous-couche visqueuse et la mesure de frottement. Ce vélocimètre est réalisé par les techniques de l'optique intégrée et possède une optique de focalisation permettant d'obtenir des volumes de mesure comparables à ce qui se fait de plus petit sans augmentation toutefois de la taille du vélocimètre. Ce capteur ainsi que la technique de fabrication utilisée sont décrits dans la première partie de cet article. Les caractérisations optiques de l'optique de focalisation, à savoir des miroirs intégrés, et des vélocimètres sont présentés dans la seconde partie de ce travail.

\section{DESCRIPTION DU VÉLOCIMÈTRE INTÉGRÉ ET DE SA CHAÎNE DE MESURE}

\section{II.1 Description générale}

L'ensemble de la chaîne de mesure associé au 
vélocimètre intégré est illustré à la figure 1. Une diode laser de longueur d'onde $830 \mathrm{~nm}$ couplée à une fibre optique monomode sert de source lumineuse pour la tête du capteur. Cette dernière, dont le fonctionnement sera décrit plus amplement dans le paragraphe suivant, est installée au sein de la paroi de telle façon qu'elle soit affleurante. Elle joue le rôle de séparateur de faisceaux optique et de collimateur. Le volume de mesure ainsi produit est localisé dans le fluide à une distance moyenne se situant entre 0 et 500 microns de l'arête de sortie de la plaquette de verre. La collection des bouffées Doppler est assurée par une fibre optique multimode. Celle-ci est collée sur la face supérieure du substrat de verre et centrée par rapport aux deux faisceaux d'émission. Les signaux optiques sont détectés par une diode avalanche en $\mathrm{Si}$ avant d'être traités par une électronique du commerce.

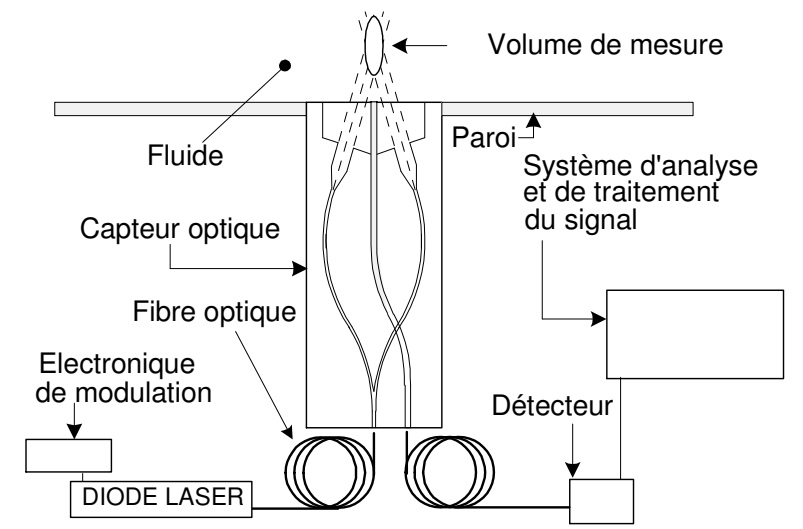

Fig. 1 Schéma de l'ensemble de la chaîne de mesure

Notre objectif est donc d'utiliser l'optique intégrée sur verre pour produire un petit volume de mesure localisé avec précision et ainsi faciliter les mesures près de la paroi. La réception se fait en rétro-diffusion, ce qui élimine toutes les réflexions parasites. De plus, le capteur étant petit, il est placé au sein de la paroi dont il devient solidaire. Il ne peut alors plus y avoir de mouvement du volume de mesure par rapport à la paroi, éliminant toute incertitude venant des phénomènes vibratoires. Ce capteur intégré présente aussi l'avantage de ne nécessiter aucun ajustement de la part de l'utilisateur. En contrepartie, mentionnons que ce vélocimètre possède le défaut de ses qualités, à savoir que la position du volume de mesure est fixée lors de l'élaboration du composant, tout comme l'interfrange, et qu'ils ne peuvent pas être modifiés ultérieurement. Enfin, comme il fonctionne en rétro-diffusion et qu'il est simplement affleurant au fluide, aucune fenêtre n'est nécessaire dans la paroi. Il suffit de pratiquer une ouverture pour y insérer le capteur.

\subsection{Description de la tête de mesure}

La partie essentielle et originale du VLDI est le composant d'émission des franges d'interférence. Celui- ci est réalisé sur un substrat de verre par échange d'ions. Sa structure est illustrée à la figure 2. La lumière provenant de la fibre optique monomode (reliée à la diode laser) est couplée au guide d'entrée du composant en verre. La largeur de ce dernier est de $1 \mu \mathrm{m}$. Ce guide est monomode à la longueur d'onde du vélocimètre, soit $830 \mathrm{~nm}$. La lumière est ensuite divisée en deux parties égales grâce à ce que l'on nomme une jonction Y. Celleci est simplement constituée du guide d'entrée et de 2 guides de sorties. Si l'angle entre les deux guides est faible (c'est-à-dire de l'ordre de $1^{\circ}$ ou moins), alors on montre que la lumière guidée s'y sépare en deux parties égales avec des pertes minimes (pertes optiques inférieures à $1 \%$ ). Les deux faisceaux optiques ainsi obtenus sont cohérents entre-eux et se propagent dans les deux bras du composant. Ils sont ensuite dirigés vers des miroirs intégrés, également réalisés par échange ionique. Ceux-ci sont de forme elliptique et font converger la lumière à leur sortie, même à l'extérieur du composant en verre. Cette convergence est essentielle pour obtenir un volume de mesure le plus petit possible et également pour avoir des interfranges régulières à l'intérieur de celui-ci.

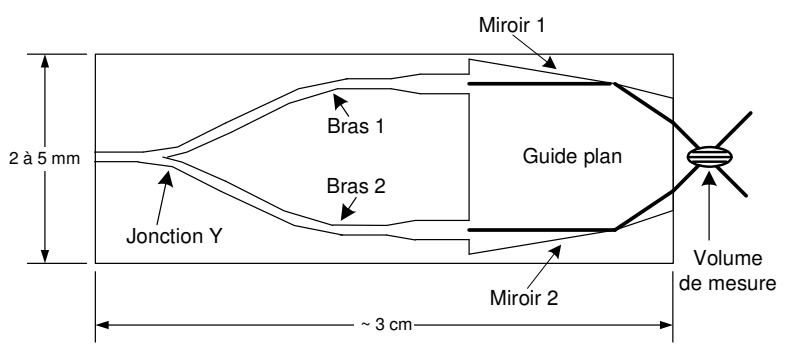

Fig. 2 Schéma de principe de la structure intégrée sur verre.

Une photographie d'un composant ainsi réalisé est illustrée à la figure 3 . Sur cette image, on distingue le substrat de verre sur lequel est réalisé la structure d'émission, la connectique nécessaire au couplage de la fibre monomode d'injection et également la fibre de collection des signaux optiques Doppler rétro-diffusés.

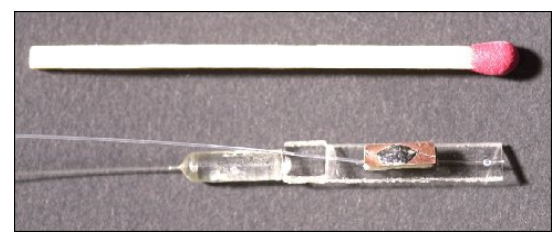

Fig. 3 Photographie de la tête d'émission intégrée ainsi que de la fibre de réception

Les miroirs intégrés fonctionnent sur le même principe physique que celui du guidage optique : la réflexion totale interne. L'échange ionique ayant pour effet d'augmenter l'indice de réfraction du verre, la zone située avant les miroirs, i.e. la zone constituant le guide plan, est échangée en même temps que les guides alors 
que celle située après les miroirs ne l'est pas. Si les angles des rayons lumineux arrivant à cette interface sont supérieurs à l'angle critique (les angles sont mesurés par rapport à la normale de l'interface), alors la lumière est entièrement réfléchie. C'est ce qui se produit pour ce composant.

Quant à elle, la convergence des faisceaux est obtenue par la forme elliptique des miroirs. En effet, en optique géométrique, c'est un fait bien connu que tous les rayons passant par un des foyers d'une ellipse passeront par le second foyer après réflexion sur l'ellipse en question. Pour notre structure, le premier foyer d'un miroir elliptique est situé à la sortie d'un des bras de l'interféromètre. Le second foyer correspond à l'emplacement du volume de mesure. La déviation des faisceaux optiques causée par le changement de milieu est prise en compte dans la conception des vélocimètres intégrés. Des simulations numériques ont été menées afin de valider cette idée. Ces simulations numériques font essentiellement appel à la propagation d'ondes et en ce sens donnent des résultats très proches de la réalité. Un exemple de résultats de simulations est illustré à la figure 4.
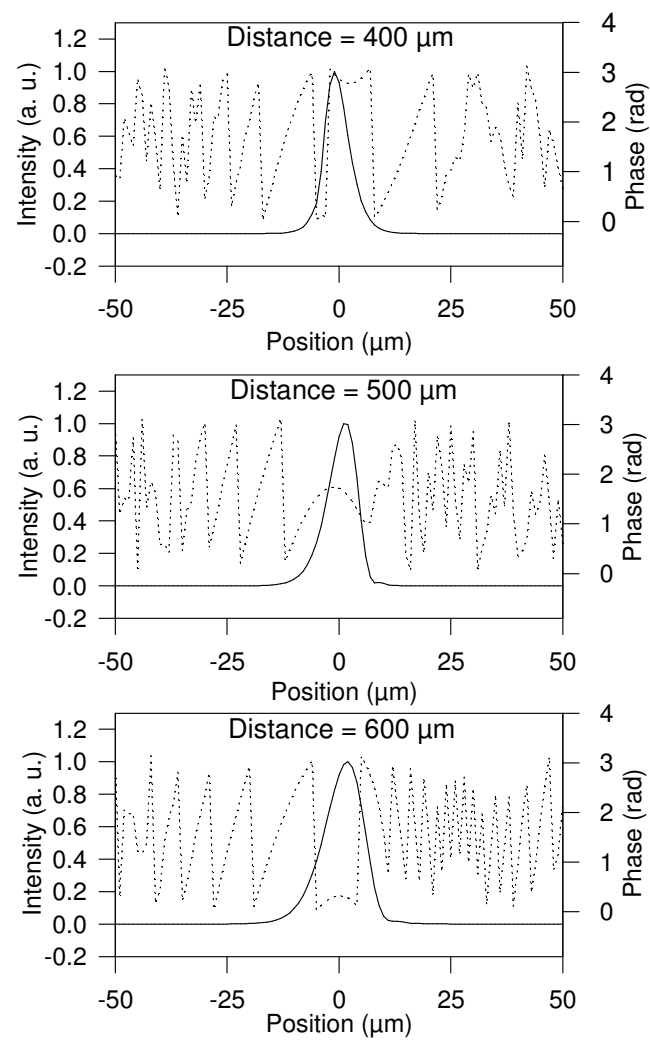

Fig. 4 Résultats de simulations numériques montrant l'intensité optique à différentes positions dans le fluide après réflexion sur un miroir intégré elliptique. Le plan focal correspond à la distance $500 \mu \mathrm{m}$.

Les simulations numériques montrent qu'il est possible de cette façon d'obtenir une largeur de faisceau de 12 $\mu \mathrm{m}$, à 1/e2 de l'amplitude maximale. Deux points sur la figure 4 montrent qu'il y a bel et bien convergence de la lumière. Le premier, c'est la phase du faisceau qui est d'abord concave puis convexe après le point focal. Le second c'est l'asymétrie du faisceau qui s'inverse avant et après le point focal et qui s'annule presque entièrement au point focal.

\subsection{Technique de l'échange d'ions}

La technique de fabrication de guides par échange d'ions repose sur la photolithographie, procédé abondamment utilisé en microélectronique. Ce procédé a pour but de transférer un motif quelconque d'un masque vers un échantillon.

La première étape consiste à nettoyer les substrats de verre. L'étape suivante est le dépôt d'une couche mince qui servira de masque aux ions lors de l'échange ionique proprement dit. En général celle-ci est de l'aluminium déposée par évaporation. L'épaisseur de la couche est de $200 \mathrm{~nm}$ environ. La troisième étape consiste à déposer à la tournette une mince couche de photorésine, matériau sensible aux rayonnements ultraviolets. La résine ayant été exposée aux rayons ultraviolets est ensuite dissoute dans une solution appropriée, alors que la résine non insolée ne sera pas enlevée par ce produit. Le masque est ainsi reproduit sur la résine. La couche masquante déposée à l'étape 2 est ensuite gravée par une solution chimique appropriée. Seules les parties non recouvertes de résine seront attaquées. Le motif initial du masque est maintenant reproduit sur l'aluminium. Après la gravure, la résine demeurant sur l'échantillon est enlevée grâce à une solution chimique. Toutes ces étapes doivent impérativement être réalisées en salle blanche. Ce n'est pas le cas pour les suivantes.

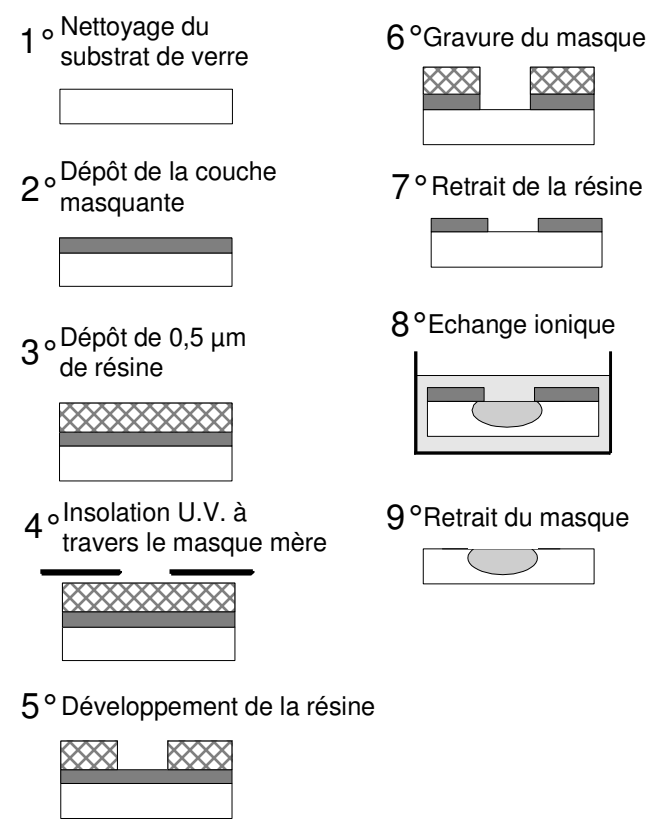

Fig. 5 Résumé des étapes de réalisation d'un guide d'onde fait par échange d'ions dans le verre. 
L'étape suivante est l'échange ionique. Il consiste à immerger la plaquette de verre dans un sel d'ions fondu. Pour un échange $\mathrm{Ag}+$ « $\mathrm{Na}+$, le sel est du AgNO3. Les ions $\mathrm{Na}+$ contenus à l'origine dans le verre vont migrer dans le sel et seront remplacés par les ions $\mathrm{Ag}+$. Ceci se produit uniquement en dessous des ouvertures pratiquées dans le masque d'aluminium. De cette manière, la structure chimique du verre est modifiée selon le design du masque d'origine. Comme cette modification de structure se traduit par une augmentation de l'indice de réfraction, des guides d'onde ayant la géométrie que l'on veut sont ainsi créés. L'avant-dernière étape du processus consiste à enlever la couche qui a servi de masque lors de l'étape précédente. Enfin, l'ultime étape de la réalisation des composants consiste à découper à la scie diamantée les motifs qui nous intéressent et à polir les arêtes d'entrée et de sortie des guides. La figure 5 schématise et résume l'ensemble de ces étapes technologiques.

\section{CARACTÉRISATIONS DES VÉLOCIMÈTRES}

\subsection{Convergence des miroirs}

Les miroirs intégrés, qui sont une structure nouvelle en optique intégrée[16], ont d'abord été testés seuls afin de vérifier expérimentalement leur pouvoir de convergence. Pour cela, des miroirs elliptiques pour lesquels la direction générale de propagation de la lumière est perpendiculaire à l'arête de sortie du verre ont été fabriqués. La lumière provenant de la diode laser connectorisée à la fibre optique monomode est couplée au guide d'entrée de ces structures de test. La longueur d'onde du laser est de $0,83 \mu \mathrm{m}$. L'arête de sortie d'un composant est imagée sur une caméra CCD Le profil d'intensité et la largeur à $1 / \mathrm{e} 2$ de l'intensité maximale ont ainsi été mesurés. Afin de visualiser l'évolution du champ après la réflexion, l'arête de sortie a été coupée et polie à plusieurs endroits.
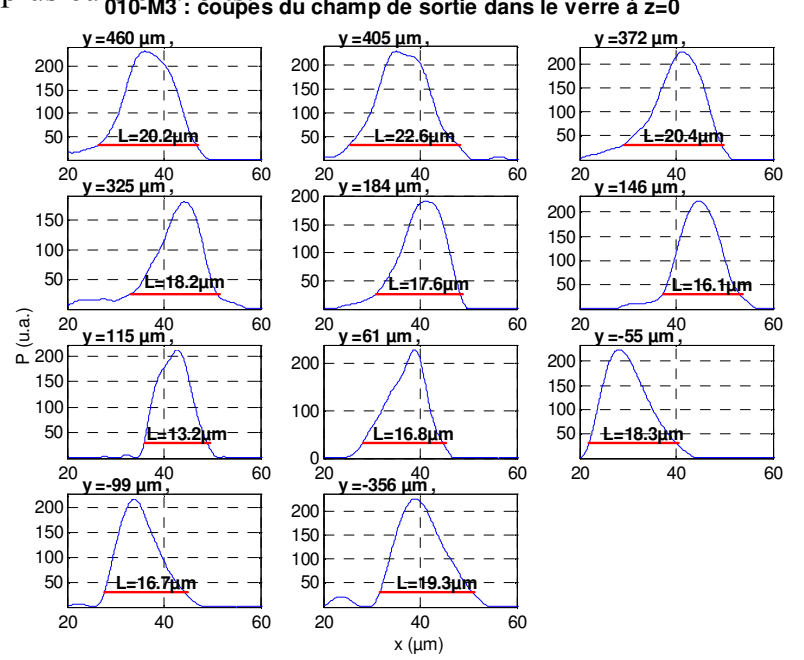

Fig. 6 Exemple de profils d'intensité mesurés sur l'arête de sortie du composant avec la caméra CCD pour différentes positions de cette arête de sortie (valeur y).
Des profils semblables à ceux représentés à la figure 6 ont été obtenus. On voit clairement qu'ils sont asymétriques et que l'asymétrie est inversée après une certaine position, tout comme nous l'avions observé par simulations numériques. Cette dernière est le point du foyer.

L'évolution de la largeur du faisceau optique après réflexion sur un miroir intégré en fonction de la distance de propagation est illustrée à la figure 7 . Pour obtenir ces deux figures, nous avons effectué deux expériences légèrement différentes. La première courbe, quant à elle, est obtenue à partir des profils d'intensité de l'arête de sortie du composant présentés à la figure 6. Pour la seconde, le composant a déjà été coupé et poli à une position en amont du point focal lors de l'expérience précédente. Un objectif de très faible profondeur de champ ( 2 à $3 \mu \mathrm{m}$ maximum) est éloigné progressivement du composant intégré de telle sorte que plusieurs plans de l'espace situés dans l'air après cette arête soient imagés sur la caméra CCD. (La distance arête du composant à la caméra CCD est fixe et grande par rapport au déplacement de l'objectif.) De cette manière, nous visualisons l'évolution du profil d'intensité telle qu'elle se produit réellement dans l'air après la réflexion sur le miroir intégré.

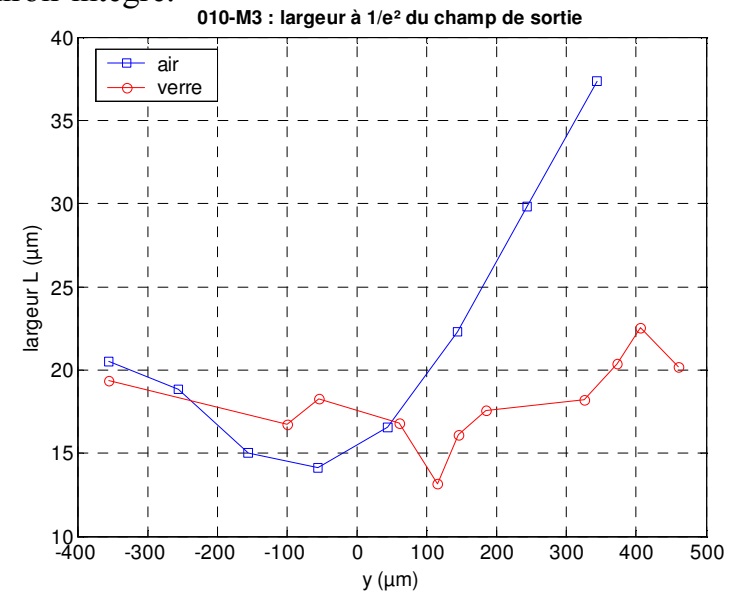

Fig. 7 Evolution de la largeur du faisceau à $1 / \mathrm{e}^{2}$ de l'intensité maximale. Pour la première courbe, le composant est coupé à plusieurs endroits et l'arête de sortie imagée sur la caméra CCD. Pour la seconde courbe, différents plans de l'espace après l'arête de sortie sont imagés sur la caméra.

A partir de la figure 7, de nouveau, nous voyons clairement l'effet de focalisation des miroirs. Le plus petit waist mesuré est de $13 \mu \mathrm{m}$ qui doit être comparé à la valeur obtenue par simulation numérique : $12 \mu \mathrm{m}$. On voit également que les waists des faisceaux mesurés des deux manières décrites précédemment sont très similaires mais que la distance focale est cependant différente. Ceci s'explique aisément par la différence d'indice de réfraction du milieu "extérieur", à savoir le verre et l'air. Plus l'indice de réfraction est voisin de celui du verre, plus loin sera situé le point focal. (Pour l'eau, le point de 
focalisation serait situé autour de la coordonnée 0 , selon les simulations numériques.) Tous ces résultats expérimentaux sont en bon accord avec les simulations numériques faites.

\subsection{Caractérisations optiques des volumes de mesures}

Des composants contenant cette fois-ci la structure d'émission des vélocimètres intégrés ont ensuite été réalisés. Tout comme pour les miroirs seuls, deux caractérisations optiques différentes ont été faites : l'une par polissages successifs de l'arête de sortie du composant, l'autre par la numérisation d'images en différents plans de l'espace.

Pour le premier type de caractérisation, la figure 8 montre un exemple des images enregistrées au centre du volume de mesure ainsi que le profil d'intensité correspondant. Le fait que les franges d'interférence soient régulières indique que la phase optique des faisceaux est bel et bien constante au point focal des miroirs. L'interfrange de ce composant est de $0,8 \mu \mathrm{m}$ et est constant à l'intérieur du volume de mesure. Ceci est évidemment un paramètre important puisqu'il conditionne la précision du vélocimètre intégré. Pour tous les échantillons testés, la visibilité des franges est toujours voisine de 1 .
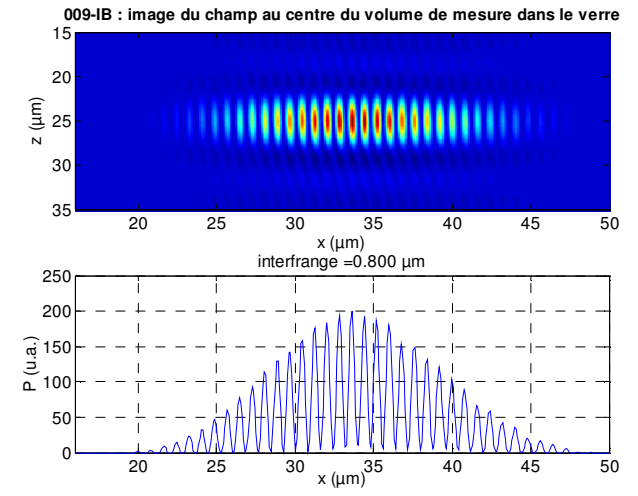

Fig. 8 Haut) Photo en pseudo-couleur du profil d'intensité au centre du volume de mesure mesuré sur l'arête de sortie du composant. Bas) Profil d'intensité correspondant.

Pour le second type de caractérisation, l'arête de sortie du composant intégré a été polie à sa position finale. L'évolution du volume de mesure dans l'air a été enregistrée. Un exemple d'images et de profils d'intensité obtenus est donné à la figure 9 . Pour ce composant, la taille du volume de mesure est de : $22 \times 33 \times 10 \mu \mathrm{m}^{3}$ à $1 / \mathrm{e}^{2}$ de l'intensité maximale.

\section{9-IB : coupes du champ de sortie de dans l'air à $z=0$ et Pin=cte}
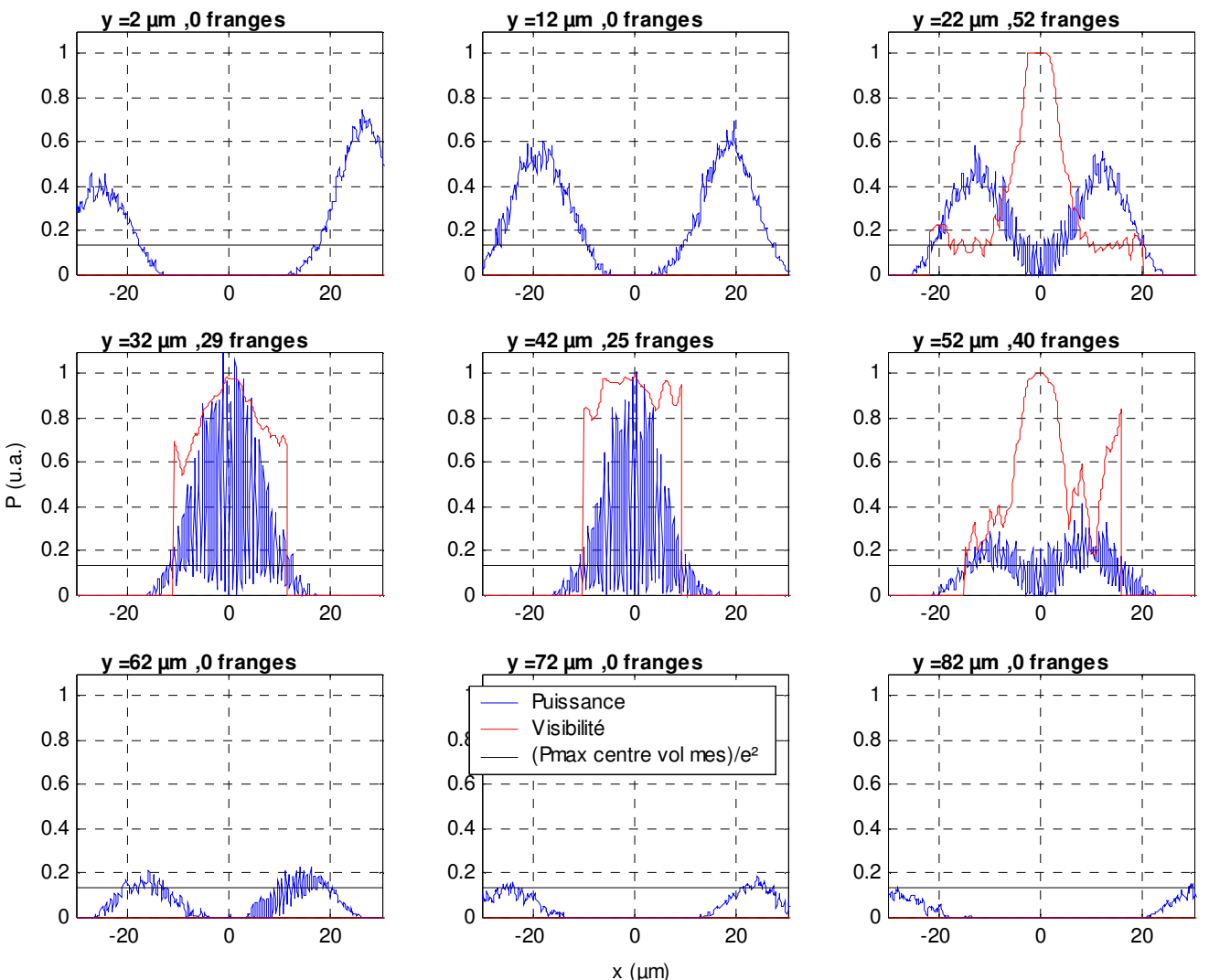

Fig. 9 Profils d'intensité mesurés à différentes positions dans l'air. Est également représentée sur ces graphiques une estimation de la visibilité des franges ainsi que le niveau à $1 / \mathrm{e}^{2}$ de l'intensité maximale. Le volume de mesure fait $22 \times 33 \times 10 \mu \mathrm{m}^{3}$ à $1 / \mathrm{e}^{2}$ et contient 25 franges au centre. 


\subsection{Résultats préliminaires des vélocimètres}

Les vélocimètres produits ont ensuite été testé sur un écoulement en air contenant des bulles d'eau. L'écoulement n'est pas très bien maîtrisé puisqu'il s'agit de la sortie d'un humidificateur à ultra-sons du commerce. L'objectif n'était pas de vérifier la précision $\mathrm{du}$ capteur mais uniquement d'obtenir des signaux Doppler et de vérifier que leur contraste et leur amplitude sont suffisants pour faire la mesure. La taille moyenne de ces bulles n'a pas été précisément mesurée mais est toutefois estimée à une dizaine de microns au maximum. Quant à elle, la vitesse de l'écoulement se situe entre 20 et $50 \mathrm{~cm} / \mathrm{s}$ selon l'écart entre le capteur et la sortie de l'humidificateur.

Afin de réaliser ces mesures, une fibre optique multimode d'ouverture numérique 0,48 a été collée au dessus du substrat de verre. Une cale, également en verre, permet d'incliner cette fibre de réception d'une angle de $25^{\circ}$. De cette façon, une plus grande quantité de lumière provenant du volume de mesure et diffusée par les particules entre dans la fibre.

Un exemple de signaux obtenu est illustré à l'image supérieure de la figure 10 L'interfrange du vélocimètre utilisée est de 1,06 $\mu \mathrm{m}$. La fréquence Doppler est nettement visible sur ce signal, représentatif des signaux reçus. En éliminant la composante basse fréquence ainsi que les fréquences hautes, on obtient un signal similaire à celui illustré à l'image inférieure de la figure 10. La vitesse mesurée est de $34,5 \mathrm{~cm} / \mathrm{s}$, ce qui correspond bien à la gamme de valeur attendue. Ces premiers résultats valident le fonctionnement de ce type de capteur intégré.
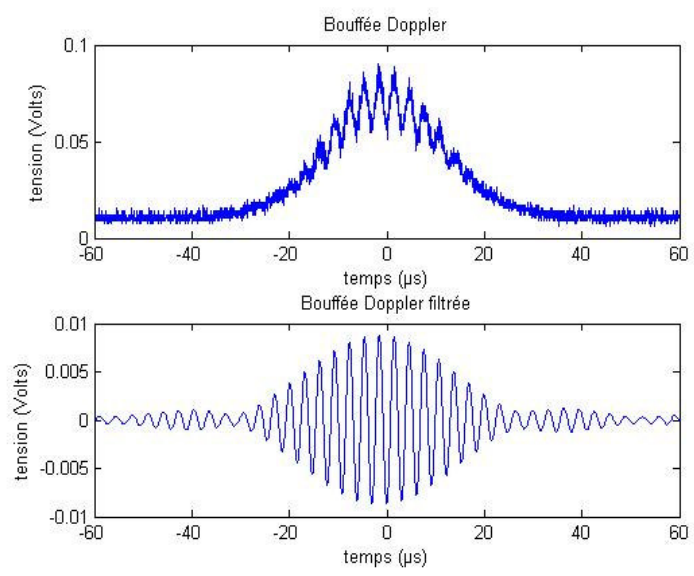

Fig. 10 Exemple de signaux Doppler obtenu avec un vélocimètre intégré dont l'interfrange vaut $0,8 \mu \mathrm{m}$. Haut) Signal Doppler brut. Bas) Signal obtenu après filtrage avec un filtre passe-bande.

\section{CONCLUSION ET PERSPECTIVES}

Un nouveau type de vélocimètre intégré a été présenté dans cet article. Il est réalisé par échange d'ions dans du verre et mesure de l'ordre de 2 à $3 \mathrm{~mm}$ de large par 2 à 3 $\mathrm{cm}$ de long. Il intègre l'optique nécessaire à la production du volume de mesure ainsi que celle servant à récupérer les signaux Doppler. Il est déporté de sa source de lumière : une diode laser et de son détecteur optique : une diode à avalanche. Il est destiné à être monté affleurant à une paroi. De ce fait, il est insensible à toutes formes de vibrations. Il mesure une composante de la vitesse dans la sous-couche visqueuse. Les points intéressants de ce capteur sont les suivants : le volume de mesure est très petit mais contient tout de même beaucoup de franges (entre 20 et 40 selon les cas), la position de ce volume de mesure est précisément connue, il fonctionne en rétrodiffusion, ce qui élimine tous les problèmes de réflexions parasites sur la paroi. Puisque la vitesse et la distance de mesure sont connues, on peut donc avoir accès au frottement entre le fluide et la paroi. Enfin il ne nécessite aucun ajustement optique de la part de l'utilisateur. De plus, de part sa conception, il peut tout aussi bien fonctionner dans des micro-canaux.

Des caractérisations optiques d'une partie essentielle de ce capteur, à savoir le miroir intégré, ont été présentées. Les résultats de mesure concordent avec les prédictions numériques. L'ensemble de la tête d'émission a également été réalisé et qualifié optiquement. Encore une fois, expérience et prédictions numériques sont en bon accord. De plus, les premiers essais en écoulement démontrent le bon fonctionnement des vélocimètres. Il reste à les caractériser complètement en soufflerie et dans une section test d'un écoulement en eau. Toutefois, de telles mesures ont été faites avec un vélocimètre similaire, mais possédant une moins bonne résolution spatiale[15]. La précisions étaient alors de 1 à $4 \%$. Nous nous attendons cette fois-ci à une précision meilleure à $1 \%$ avec la nouvelle génération.

\section{REFERENCES}

[1] Hanratty TH.J., Campbell J.A., 1983, "Chapter 11 : Measurement of wall shear stress", in Fluid Mechanics Measurements, Golstein R.J. Ed., Hemisphere Publishing Corp., distributed by Springer-Verlag, pp. 559-611.

[2] Saetran L.R., 1987, "Comparison of five methods for determination of the wall shear stress, AIAA J., 25, 11, pp. 1524-1527.

[3] Gasser D., Thomann H., Dengel P., 1993, "Comparison of four methods to measure wall shear stress in a turbulent boundary layer with separation", Experiments in Fluids, 15, pp. 27-32.

[4] Fernholtz, H.H., Janke, G., Schober, M., Wagner, P.M. and Warnack, D., 1996, New developments and applications of skin-friction measuring techniques, Meas. Sci. Technol., 7, pp. 1396-1409.

[5] Martin S.R., Bates C.J., 1992, "Small-probe-volume laser Doppler anemometry measurement of turbulent flow near the wall of a rib-roughened channel" Flow Meas. Instrum. Vol. 3, No. 2, pp. 81-88.

[6] Leprince F., Riethmuller M.L., 1986, "LDV Measurements in a viscous sublayer : determination of skin friction", Laser Techniques and Applications 
in Fluids Mechanics - Proceedings of the 3rd International Symposium, Lisbon, Portugal, paper 16.

[7] Aizu Y., Ushizaka T., Asakura T., 1985, "Measurement of the Velocity Gradient Using a Laser Doppler Phenomenon", Appl. Phys. B 36, pp. 155-161.

[8] Keveloh C., Staude W., 1983, "Determination of velocity gradients with scattered light crosscorrelation measurements" Applied Optics, Vol. 22, No 2, pp. 333-338.

[9] Mishina H., Vlachos N.S., Whitelaw J.H., 1979, "Effect of wall scattering on SNR in laser Doppler anemometry", Applied Optics, Vol. 18, No 14, pp. 2480-2485.

[10] Cline C., Deutsh S., 1993, "On elevated RMS levels in wall-bounded turbulent flows when measured by laser Doppler velocimetry", Experiments in Fluids, Vol. 15, pp. 130-132.

[11] Wernet M.P., Edwards R.V., 1986, "Implementation of a new time of flight laser anemometer", Applied Optics, Vol. 25, No 5, pp. 644-648.

[12] Dybbs A., Edwards R.V., 1987, "Refractive index matching for difficult situations", 2nd Int. Conf. Laser Anemometry - Advances and Applications, Glascow, UK, BHRA, Cranfield, paper 1-22.

[13] Durst F., Keck T., Kleine R., 1985, "Turbulence quantities and Reynolds stress in pipe flow of polymer solutions", 1st Int. Conf. Laser Anemometry - Advances and Applications, Manchester, UK, BHRA, Cranfield, paper 35-52.

[14] Lemaittre-Auger, P., Cartellier, A., Benech, P. et Schanen Duport, I., ${ }^{2}$ Integrated Laser Doppler Velocimeter made by Ion-Exchanged in Glass Substrate $^{2}$, Developments in Laser Techniques to Fluid Mechanics and Applications, Selected Papers from the 8th International Symposium, SpringerVerlag, editors : Adrian, R.J. et al., pp. 39-51, 1997.

[15] Lemaître-Auger, P., Hasegan, L., Cartellier, A., Benech, P. and Schanen Duport, I., ${ }^{2}$ Integrated laser Doppler velocimeter for wall friction measurements : new prototypes and performance", 9th International Symposium on Applications of Laser Techniques to Fluid Mechanics, Lisbon, Portugal, paper \# 238, July 13-16 1998.

[16] P. Lemaître-Auger, S. Gluck, D. Bucci, A. Cartellier, $\mathrm{P}$. Benech, « Focusing integrated mirrors realized by silver ion-exchange : application to laser Doppler velocimeter with ultra-high spatial resolution "), Proceedings of the SPIE 5728, Photonics West 2005, San Jose CA, papier 5728-02 (à paraître). 